\title{
Coexistent Papillary Thyroid Carcinoma and Its Anaplastic Transformation in Cervical Lymph Node Metastasis
}

\author{
Seulki Song', Dong-Han Lee ${ }^{2}$, Hyojin $\mathrm{Kim}^{3}$ and Woo-Jin Jeong ${ }^{2}$ \\ Department of Otorhinolaryngology-Head and Neck Surgery, Gyeongsang National University College of Medicine, Gyeongsang \\ National University Changwon Hospital, ${ }^{1}$ Changwon, Department of Otorhinolaryngology-Head and Neck Surgery, Seoul National \\ University College of Medicine, Seoul National University Bundang Hospital', Seongnam, Department of Pathology, Seoul National \\ University College of Medicine, Seoul National University Bundang Hospital, Seongnam, Korea
}

The transformation of papillary thyroid carcinoma (PTC) to anaplastic thyroid carcinoma (ATC) is well documented in the literature but is an exceptionally rare occurrence in metastatic foci outside the primary thyroid lesion. Even rarer is the simultaneous occurrence of PTC and ATC in the cervical lymph nodes. We report the case of an 85-year-old man who presented with a rapidly growing neck mass diagnosed as PTC. Following surgery, multiple ATC foci in the metastatic cervical lymph node were found coexisting with PTC, whereas in the thyroid, only PTC was found. This case is of high clinical significance because transformation of PTC to ATC outside the thyroid gland per se is very rare and because it suggests rapidly growing tumors in an elderly patient. The use of core needle biopsies in cases with suspected rapid tumor growth can aid in proper diagnosis, surgical decision making, and patient counselling.

Key Words: Thyroid cancer, Papillary thyroid carcinoma, Lymphatic metastasis, Anaplastic transformation

\section{Introduction}

Anaplastic thyroid carcinoma (ATC) is rare and accounts for only $5 \%$ of all thyroid cancers. However, it is the most aggressive form of thyroid cancer and has a very poor prognosis. ${ }^{1)}$ Evidence that ATC arises from differentiated thyroid carcinoma is accumulating. ${ }^{2)}$ However, the mechanism of transformation has yet to be clarified. In almost all cases, ATC arises within the thyroid gland and seldom occurs in extrathyroidal foci. ${ }^{3-13)}$ Transformation of a metastatic differentiated thyroid carcinoma to ATC in the cervical lymph node is extremely rare. ${ }^{6,8,9,13)}$ We report a case of ATC in the lateral cervical lymph node coexisting with papil- lary thyroid carcinoma (PTC) at the time of surgery which was suggestive on the initial core needle biopsy of the lymph node.

\section{Case Report}

An 85-year-old man presented with a rapidly growing mass in the left neck, which was detected just one week prior. An initial computed tomography (CT) scan of the neck revealed conglomerate or multiple lymphadenopathy, with the largest node measuring up to $4.9 \mathrm{~cm}$ and showing necrotic portions and perilesional infiltration at left levels IV, VI, and the anterior mediastinum, suggesting metastatic lymphadenopathy (Fig. 1A, B). Ultrasonography of the thyroid revealed a high-

Received April 10, 2020 / Revised May 12, 2020 / Accepted May 12, 2020

Correspondence: Woo-Jin Jeong, MD, PhD, Department of Otorhinolaryngology-Head and Neck Surgery, Seoul National University Bundang Hospital, 82, Gumi-ro 173 Beon-gil, Bundang-gu, Seongnam 13620, Korea

Tel: 82-31-787-7407, Fax: 82-31-787-4057, E-mail: safar@snubh.org

Copyright (c) the Korean Thyroid Association. All rights reserved.

(i) (\$) This is an open-access article distributed under the terms of the Creative Commons Attribution Non-Commercial License (http://creative(c), (1) \& 8 commons.org/licenses/by-nc/4.0/), which permits unrestricted non-commercial use, distribution, and reproduction in any medium, provided the original work is properly cited. 

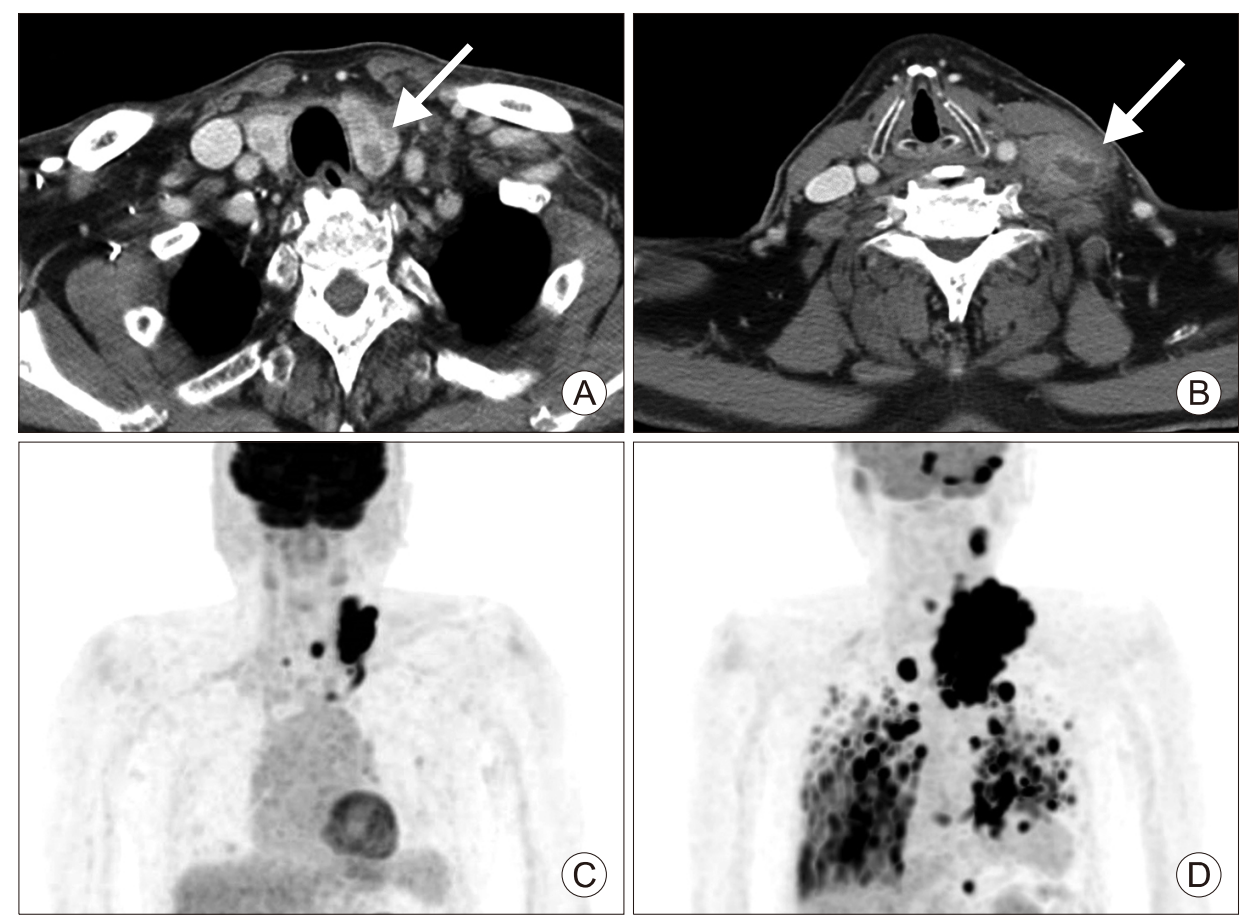

Fig. 1. (A) Computed tomography of the neck shows a large-sized mass $(2 \times 2 \times 3.2 \mathrm{~cm})$ in the left thyroid gland (arrow). (B) Computed tomography of the neck shows the left level IV lymph node with enlarged size $(4.7 \times 3.1 \mathrm{~cm})(\mathrm{arrow})$. (C) Positron emission tomography-computed tomography (PET-CT) scan. Hypermetabolic nodule in the left thyroid lobe. Hypermetabolic enlarged lymph nodes in left neck level III, IV, right level VI and superior mediastinum. Small nodules with hypermetabolism in both lungs. (D) PET-CT scan. Local recurrence with disseminated metastasis to lymph nodes, lungs, bones, cerebellum, left adrenal gland, and cardiac RV wall.

ly suspicious nodule (K-TIRADS ${ }^{14)}$ category 5 , L1) of $1.8 \mathrm{~cm}$ in size in the left lower thyroid gland with hypoechoic, solid, irregular, parallel, and spiculated features together with microcalcification, void vascularity, and extrathyroidal extension into the infrahyoid strap muscles. Core needle biopsy was commenced for the L1 lesion and the left large level IV lymph node. On pathologic examination, PTC with no other discernable features was found in the L1 lesion. However, malignant neoplasm was diagnosed in the lymph node specimen with a minor PTC component; the majority of the biopsied lymph node specimen revealed poorly differentiated histology different from PTC (Fig. 2A). Immunohistochemistry was performed for the differential diagnosis. Cytokeratin, thyroid transcription factor-1 (TTF-1), and paired box gene 8 (PAX8) were negative (Fig. 2B); only vimentin was expressed in the poorly differentiated component. The finding could be suggestive of both anaplastic transformation from PTC and collision tumor composed of PTC and sarcoma.
Further work-up revealed multiple, small metastatic foci in the lung, which were considered metastases of PTC at the time of diagnosis. Postoperative radioiodine ablation was planned to address the lesions (Fig. 1C).

The initial assessment in this elderly man was PTC of the left thyroid gland, with multiple lateral cervical lymph node metastases and some extension into the anterior mediastinum; possible PTC metastasis to the lung was to be addressed with radioactive iodine therapy following surgery. A total thyroidectomy with modified radical neck dissection of the left neck (levels Ila, III, IV, Va, Vb, VI, and sternocleidomastoid muscle) and anterior superior mediastinal lymph nodes was carried out. The left recurrent laryngeal nerve was adherent to the thyroid gland; however, the tumor could be isolated without damage to the nerve. The tumor was severely adhered to the internal jugular vein, and tumor emboli were visible within the vessel, which had to be ligated and resected. The sternocleidomastoid muscle was also invaded. The carotid artery was 

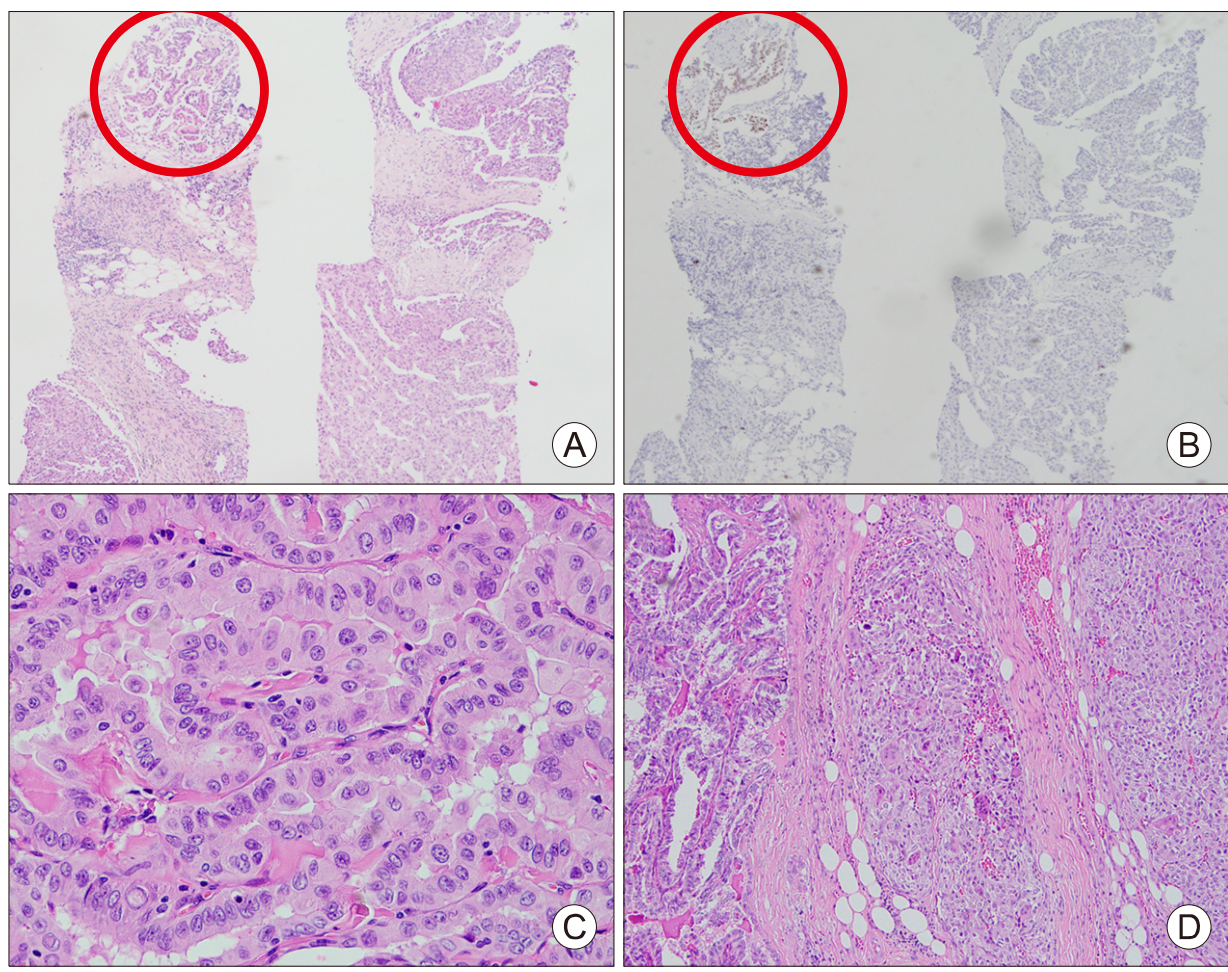

Fig. 2. Pathology finding of cervical lymph node core needle biopsy specimen. (A) The metastatic tumor composed of a minor papillary thyroid carcinoma component (left upper, red circle) and the majority of the biopsied specimen with poorly differentiated histology different from PTC; Hematoxylin \& Eosin staining (H\&E) $\times 40$. (B) Immunohistochemical staining of PAX8. PAX8 was expressed only in the papillary thyroid carcinoma component (left upper, red circle) and negative in the poorly differentiated tumor component; $H \& E \times 40$. Pathology finding of surgically resected specimen. (C) Thyroid; H\&E $\times 400$. (D) Lymph node; H\&E $\times 400$.

preserved. The thyroid and the cervical specimen was gross totally resected; however, gross extranodal extension (ENE) of the tumor was present, and some tumor spillage was inevitable, resulting in R1 resection.

The patient recovered without obvious complications and was discharged on postoperative day 9 . Final pathology revealed conventional PTC within the thyroid gland with an adequate safety margin (Fig. 2C). However, PTC and ATC were co-existent in the cervical lymph node (Fig. 2D). The majority of the metastatic tumor revealed an ATC component, and the focal area of the ATC was positive for PAX8. After review of the final pathology report and multidisciplinary tumor board discussion, postoperative radiotherapy was recommended. However, due to the advanced age and poor performance status, the patient and his family refused further adjuvant therapy. Two months postoperatively, he presented to the emergency room with dyspnea. A neck CT scan revealed a large mass in the left, previously dissected neck. A core needle biopsy under ultrasonography guidance was obtained and recurrent ATC was confirmed. Whole-body positron emission tomography-CT (PET-CT) revealed disseminated metastatic disease (Fig. 1D). The patient refused further treatment and died of the disease 10 weeks following initial surgery.

\section{Discussion}

More than $90 \%$ of thyroid cancers are differentiated thyroid carcinomas and most have an excellent prognosis. ${ }^{15)}$ In striking contrast, more than half of ATCs present with extensive extrathyroidal extension, lymph node metastasis, and distant disease at presentation. The disease-specific mortality for ATC exceeds well over $90 \%$.

Although the majority of ATC originate from differentiated carcinomas within the thyroid gland, trans- 


\section{Anaplastic Transformation of Papillary Thyroid Carcinoma in Cervical Lymph Node}

formation of metastatic differentiated thyroid carcinomas into ATC has been reported. ${ }^{3-13)}$ Among these anecdotal case reports, four reports with a total of ten patients identified ATC transformed from PTC within the cervical lymph node (Table 1). ${ }^{6,8,9,13)}$ Six of the patients were diagnosed with ATC in the metastatic lesions of cervical lymph nodes after thyroidectomy; in four cases, ATC was found simultaneously with PTC in the cervical lymph node metastasis, analogous to our present case. Seven case reports documented ATC in distant metastatic lesions. ${ }^{3,7,10-12)}$ The mechanism of anaplastic transformation in the well-differentiated thyroid carcinoma is still unclear. Overexpression of p53 and Ki-67 might be correlated with anaplastic transformation. ${ }^{10)}$ Further study is necessary to define this mechanism.

In our patient, ATC and PTC coexisted only in the core needle biopsy specimen of cervical lymph nodes. The diagnosis of ATC in lymph node biopsies was difficult, because 1) there was no evidence of ATC in the thyroid biopsy specimen, and 2) PAX8 was negative in the poorly differentiated component. PAX8 is a transcription factor that is expressed in $70-80 \%$ of ATC. In the resected lymph node specimen, PAX8 was expressed focally in the poorly differentiated component and led to the final diagnosis. Nevertheless, in retrospect, core needle biopsy provided important clinical clue regarding the aggressiveness of the otherwise in- dolent cancer in this patient, which might not have been possible with FNA.

In one study, long-term survival was more than five years in two out of five patients after surgery. ${ }^{8)}$ However, in this case, the tumor rapidly progressed within two months after the operation, despite a safe resection margin and adequate cervical lymphadenectomy. As shown in other cases, recurrent thyroid cancer that has converted in metastatic lesions is expected to show a poor prognosis similar to that of generalized ATC.

In summary, this case is a very rare case of a metastatic PTC that converted to ATC in the cervical lymph nodes. It is illustrative in that despite a diagnosis of differentiated thyroid cancer in the primary site, rapidly growing tumor in an elderly should raise an index of suspicion for more aggressive pathologies, including collision tumor of more aggressive nature or anaplastic transformation, as in this case. Furthermore, proper utilization of core needle biopsy may aid in earlier diagnosis of coexistent or transformed aggressive pathologies. The patients with anaplastic transformation have dismal prognosis. Because some cases showed good oncologic outcome after active treatment, ${ }^{8)}$ we recommend active ablation of tumor with surgery, radioactive iodine therapy, and external beam radiation therapy.

Table 1. Summary of current reported literatures on anaplastic transformation of PTC at cervical lymph nodes

\begin{tabular}{|c|c|c|c|c|c|}
\hline Author & Age/Sex & $\begin{array}{l}\text { Duration between } \\
\text { identification of primary } \\
\text { PTC and cervical ATC }\end{array}$ & $\begin{array}{c}\text { Post-operative } \\
\text { RT }\end{array}$ & $\begin{array}{l}\text { Post-operative } \\
\text { radioactive iodine }\end{array}$ & Follow-up \\
\hline Sato et al. ${ }^{6)}$ & $77 / M$ & 0 (Simultaneous) & Not given & Not given & DOD \\
\hline \multirow[t]{5}{*}{ Ito et al. ${ }^{8)}$} & $64 / M$ & 3 years & Not given & Not given & DOD \\
\hline & $80 / F$ & 0 (Simultaneous) & Not given & Not given & Alive \\
\hline & $51 / F$ & 22 years & Given & Not given & DOD \\
\hline & $79 / M$ & 6 years & Not given & Not given & DOC \\
\hline & $77 / F$ & 0 (Simultaneous) & Given & Not given & Alive \\
\hline \multirow[t]{3}{*}{ Sung et al. ${ }^{9)}$} & $64 / M$ & 39 years & Not given & Not given & DOD (4 months) \\
\hline & $63 / M$ & 7 years & Not given & Not given & DOD (3 months) \\
\hline & $27 / M$ & 8 years & Not given & Given & DOD (4 months) \\
\hline $\begin{array}{l}\text { Deutschmann } \\
\text { et al. }{ }^{13)}\end{array}$ & $60 / M$ & 0 (Simultaneous) & Given & Given & Alive for 1 year \\
\hline Present case & $85 / M$ & 0 (Simultaneous) & Not given (refused) & Not given & DOD (2 months) \\
\hline
\end{tabular}

ATC: anaplastic thyroid carcinoma, DOD: died of disease, PTC: papillary thyroid carcinoma, RT: radiation therapy 


\section{Conflicts of Interest}

No potential conflict of interest relevant to this article was reported.

\section{Orcid}

Seulki Song: https://orcid.org/0000-0001-5791-2607

Dong-Han Lee: https://orcid.org/0000-0001-9440-9744

Hyojin Kim: https://orcid.org/0000-0001-9201-8328

Woo-Jin Jeong: https://orcid.org/0000-0001-8577-6964

\section{References}

1) Nishiyama RH, Dunn EL, Thompson NW. Anaplastic spindle-cell and giant-cell tumors of the thyroid gland. Cancer 1972;30(1):113-27.

2) Papp S, Asa SL. When thyroid carcinoma goes bad: a morphological and molecular analysis. Head Neck Pathol 2015;9(1):16-23.

3) Kawahara E, Ooi A, Oda $Y$, Katsuda $S$, Terahata $S$, Michigishi T. Papillary carcinoma of the thyroid gland with anaplastic transformation in the metastatic foci. An immunohistochemical study. Acta Pathol Jpn 1986;36(6):921-7.

4) Wiseman SM, Loree TR, Rigual NR, Hicks WL Jr, Douglas WG, Anderson GR, et al. Anaplastic transformation of thyroid cancer: review of clinical, pathologic, and molecular evidence provides new insights into disease biology and future therapy. Head Neck 2003;25(8):662-70.

5) Togashi S, Oka K, Kanayama R, Koyamatsu S, Tobita T, Yatabe $\mathrm{Y}$, et al. Thyroid anaplastic carcinoma transformed from papillary carcinoma in extrathyroid area. Auris Nasus Larynx 2004;31(3):287-92.

6) Sato K, Waseda R, Tatsuzawa Y, Soma R, Ueda Y, Katsuda S. Papillary thyroid carcinoma with anaplastic transformation showing a rhabdoid phenotype solely in the cervical lymph node metastasis. Pathol Res Pract 2006;202(1):55-9.

7) Sotome K, Onishi T, Hirano A, Nakamaru M, Furukawa A, Miyazaki $\mathrm{H}$, et al. A rare case of anaplastic transformation within the metastatic site of the retroperitoneal region in a patient 17 years after total thyroidectomy for papillary carcinoma of the thyroid beginning with multiple bone metastases. Thyroid 2007;17(12):1309-11.

8) Ito $Y$, Higashiyama T, Hirokawa M, Fukushima M, Inoue $\mathrm{H}$, Yabuta $\mathrm{T}$, et al. Prognosis of patients with papillary carcinoma showing anaplastic transformation in regional lymph nodes that were curatively resected. Endocr J 2008;55(6):985-9.

9) Sung TY, Hong SW, Kang SW, Lee SC, Jeong JJ, Lee YS, et al. Anaplastic transformation of metastatic papillary thyroid carcinomas in the cervical lymph nodes: report of 3 cases. Korean J Endocr Surg 2008;8(3):210-4.

10) Takeshita $Y$, Takamura $T$, Minato $H$, Misu $H$, Ando $H$, Yamashita $\mathrm{T}$, et al. Transformation of p53-positive papillary thyroid carcinoma to anaplastic carcinoma of the liver following postoperative radioactive iodine-131 therapy. Intern Med 2008;47(19):1709-12.

11) Angeles-Angeles A, Chable-Montero F, Martinez-Benitez B, Albores-Saavedra J. Unusual metastases of papillary thyroid carcinoma: report of 2 cases. Ann Diagn Pathol 2009;13(3): 189-96.

12) Al-Qsous W, Miller ID. Anaplastic transformation in lung metastases of differentiated papillary thyroid carcinoma: an autopsy case report and review of the literature. Ann Diagn Pathol 2010;14(1):41-3.

13) Deutschmann M, Khalil M, Bhayana S, Chandarana S. Occult multifocal papillary thyroid microcarcinoma presenting as a supraclavicular mass containing anaplastic thyroid carcinoma. JAMA Otolaryngol Head Neck Surg 2013;139(4):415-8.

14) Na DG, Baek JH, Sung JY, Kim JH, Kim JK, Choi YJ, et al. Thyroid imaging reporting and data system risk stratification of thyroid nodules: categorization based on solidity and echogenicity. Thyroid 2016;26(4):562-72.

15) Albores-Saavedra J, Henson DE, Glazer E, Schwartz AM. Changing patterns in the incidence and survival of thyroid cancer with follicular phenotype-papillary, follicular, and anaplastic: a morphological and epidemiological study. Endocr Pathol 2007;18(1):1-7. 